\title{
Adaptive Multiuser MMSE Down-Link Receiver Techniques for UTRA TDD
}

\author{
I.G. Stirling ${ }^{(1)}$, D. García-Alís ${ }^{(1)}$, S. Weiss ${ }^{(2)}$, G.W. Rice ${ }^{(1)}$, R.W. Stewart ${ }^{(1)}$ \\ (1) Department of Electronic and Electrical \\ (2) Communications Research Group \\ Engineering \\ University of Strathclyde \\ Glasgow G1 1XW, SCOTLAND, U.K. \\ \{iain,daniel,garrey,bob\}@spd.eee.strath.ac.uk \\ Dept. of Electronics \& Computer Science \\ University of Southampon \\ Southampton S017 1BJ, UK \\ sw1@ecs.soton.ac.uk
}

\begin{abstract}
We present three joint adaptive mean squared error receiver structures for a time division duplex down-link scenario in which a single mobile station is allocated several code-multiplexed channels. The first structure is a bank of single channel MMSE receivers. The second is composed of an equaliser common to all the received channels and a bank of matched filters for despreading. The equaliser can be updated using a multiple-error filtered-X LMS algorithm. The final proposed structure features both an equaliser and a bank of MMSE receivers. The performance of the three architectures is tested and compared under multipath fading channel propagation conditions and BER values are provided.
\end{abstract}

\section{Introduction}

The proposed UTRA time division duplex (TDD) system [1] makes use of a superposition of two short spreading codes [2]. Although the use of orthogonal codes minimises multiple access interference (MAI), the presence of multipath destroys this orthogonality and MAI increases significantly [3]. Therefore the use of MAI suppression techniques is expected to improve the performance of such a communication system.

In synchronous spread spectrum systems using short spreading sequences, minimum mean squared error (MMSE) receivers are popular and have a number of advantages [4]. A modification of this receiver is presented in [5] which can decode signals spread with two sequences which might span over more than one symbol period. This receiver minimises MAI but it is a single receiver structure, only capable of decoding a single signal spread using an assigned pair of codes. Truly joint receivers decoding all code-multiplexed signals at once have been developed for improved receiver performance. Besides the performance advantages, in a UTRA TDD environment a single user might be assigned more than one channel. Thus, computationally inexpensive joint detection systems that are capable of decoding more than one channel are advantageous.

In this paper we focus on the scenario of a down-link UTRA TDD system, whereby several channels are to be decoded jointly in the mobile station. First, a basic introduction to UTRA TDD is given. Next, three structures for joint detection are introduced, followed by simulations carried out under multipath Rayleigh fading propagation conditions. The results obtained are then presented and finally conclusions are drawn.

\section{UTRA TDD}

Duplex communication in TDD is provided by means of time slots which separate uplink and downlink [6]. In addition to this TDMA [7] scheme, UTRA TDD includes a CDMA [8] component, whereby users are separated through the use of spreading codes within each time slot.

Two spreading codes are used in UTRA TDD to obtain the spread spectrum signal [2]. The $m$ th data symbol for the $k$ th user $d_{k}[m]$ is spread using a channelisation code $c_{\mathrm{ch}, k}$ and a scrambling code $c_{\text {ser, } k}$ which are defined as

$$
\begin{gathered}
c_{\mathrm{ch}, k}=\left[\begin{array}{llll}
c_{\mathrm{ch}, k}[0] & c_{\mathrm{ch}, k}[1] & \ldots & c_{\mathrm{ch}, k}[Q-1]
\end{array}\right]^{T} \\
c_{\mathrm{scr}}=\left[\begin{array}{llll}
c_{\mathrm{scr}, k}[0] & c_{\mathrm{scr}, k}[1] & \ldots & c_{\mathrm{srr}, k}\left[Q_{\mathrm{MAX}}-1\right]
\end{array}\right]^{T}
\end{gathered}
$$

where $Q$ is the spreading factor with possible values $Q=\{1,16\}$ and $Q_{\mathrm{MAX}}$ is the maximum value of the spreading factor. The spreading process of a symbol $d_{k}[\mathrm{~m}]$ for $Q=16$ is shown in Figure 1 .

The channelisation codes $\boldsymbol{c}_{\mathrm{ch}, k}$ are orthogonal variable spreading factor (OVSF) codes [9], and the scrambling codes $c_{\mathrm{scr}, k}$ are specified in [2]. Spread spectrum data is organised in data bursts of type $1[6]$, the structure of which is shown in Figure 2. It contains two data fields, a midamble for training purposes and a guard period (GP) to avoid overlapping of consecutive bursts. 


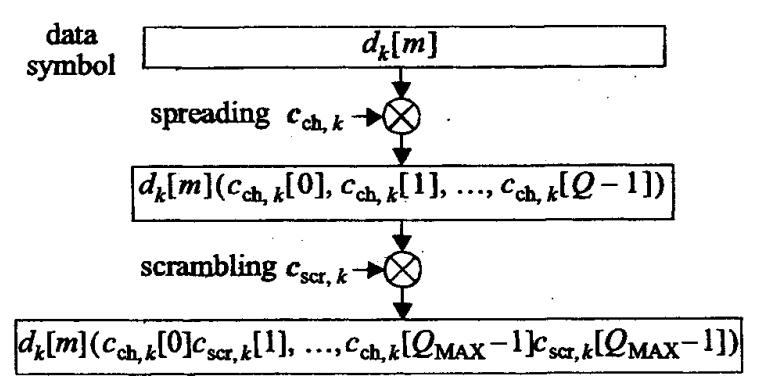

Figure 1: Spreading and scrambling for $Q=16$

\section{Joint Detection Receivers}

The three different receiver structures implemented and tested in this paper are presented next. These receivers decode a total of $K$ channels assigned to the same user.

\subsection{Bank of MMSE receivers}

The first structure tested consists of a bank of $K$ MMSE receivers [4] as shown in Figure 3. The received signal at epoch $n, x[n]$, is fed to the $K$ different branches, where it is filtered by the adaptive MMSE receivers, each of which have $N$ coefficients in a vector $\boldsymbol{w}_{k}[n]$, where it has been assumed that $N=Q_{\mathrm{MAX}}$. The outputs of the MMSE receivers are downsampled by a factor of $N$, effectively despreading the received signal and producing an estimate of the $m$ th transmitted symbol $\hat{d}_{k}[m]=\hat{d}_{k}[n N]$

$$
\hat{d}_{k}[n]=w_{k}^{H}[n] x[n]
$$

where $x[n]$ is the input samples vector

$$
x[n]=[x[n] x[n-1] \ldots x[N-1]]^{T}
$$

Using the training sequence $d_{k}[m]$ as the desired signal, the error signal is calculated as

$$
e_{k}[m]=d_{k}[m]-\hat{d}_{k}[m]
$$

A mean squared error (MSE) can be used to update the MMSE receivers' weights, the cost function to minimise being

$$
\begin{aligned}
\xi & =\mathrm{E}\left\{e_{k}^{2}[m]\right\} \\
& =\mathrm{E}\left\{\left(d_{k}[m]-w_{k}^{H}[n N] x[n N]\right)^{2}\right\}
\end{aligned}
$$

In the simulations carried out on this paper, an adaptive

\begin{tabular}{|l|l|l|}
\hline $\begin{array}{l}\text { data field } \\
976 \text { chips }\end{array}$ & $\begin{array}{l}\text { data field } \\
976 \text { chips }\end{array}$ \\
\hline & $\begin{array}{c}\text { midamble } \\
512 \text { chips }\end{array}$ \\
\hline 2560 chips
\end{tabular}

Figure 2: Data burst of type 1

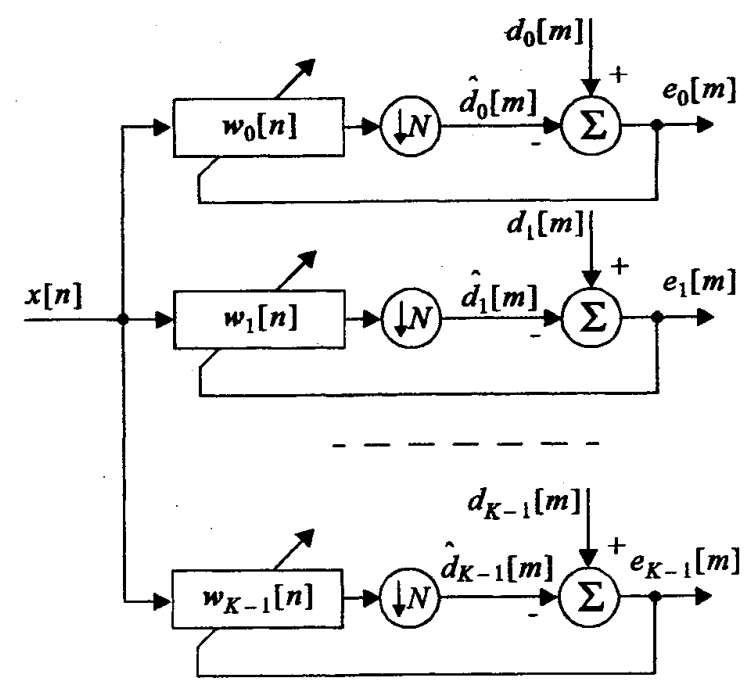

Figure 3: Bank of $K$ MMSE filters

solution is chosen which minimises the instantaneous squared error instead of the MSE. In particular, the normalised least mean squares (NLMS) algorithm [10] is used. The expression used to update the weights of the MMSE receivers of Figure 3 is

$$
w_{k}[m+1]=w_{k}[m]+2 \frac{\mu}{x^{H}[m] x[m]} e_{k}^{*}[m] x[m]
$$

where $\mu$ is the MMSE receiver step size.

\subsection{Common Equalisation}

The common equalisation joint detection setup is shown in Figure 4. It consists of an adaptive equaliser followed by a bank of filters matched to the despreading sequence of interest $c_{k}$, which decode each of the $K$ signals of interest. Under the UTRA TDD environment, the spreading code $c_{k}$ is the multiplication element by element of the channelisation and scrambling code

$c_{k}=\left[c_{\mathrm{ch}, k}[0] c_{\mathrm{scr}, k}[0] \ldots c_{\mathrm{ch}, k}\left[Q_{\mathrm{MAX}}-1\right] c_{\mathrm{scr}, k}\left[Q_{\mathrm{MAX}}-1\right]\right]^{T}(8)$

The received signal vector $x[n]$ is filtered by the $M$ tap adaptive equaliser $h[n]$, and therefore, in this case $x[n]$ is an $M$ dimensional vector

$$
x[n]=[x[n] x[n-1] \ldots x[M-1]]^{T}
$$

The output of the equaliser $y[n]$ is defined as

$$
y[n]=[y[n] y[n-1] \ldots y[n-N+1]]^{T}
$$

where

$$
y[n]=h^{H}[n] x[n]
$$




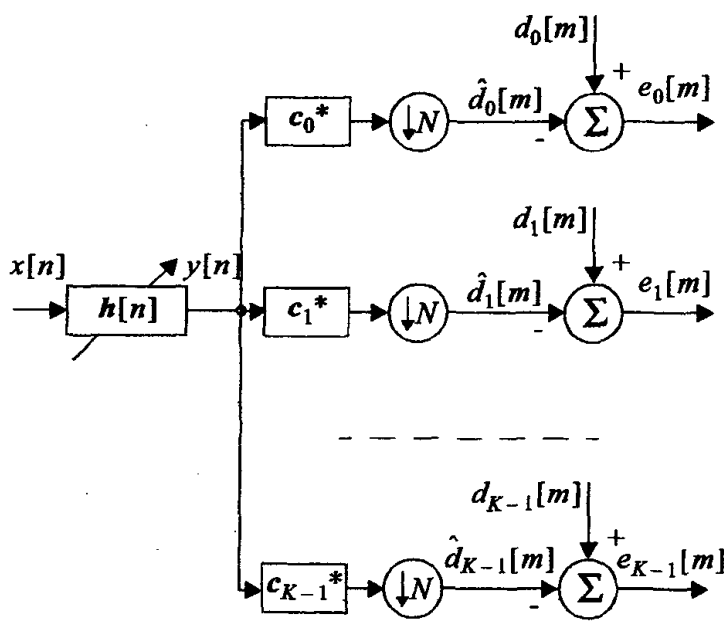

Figure 4: Common equaliser joint detection receiver

where

$$
y[n]=X^{T}[n] h^{*}[n]
$$

$$
X[n]=[x[n] x[n-1] \ldots x[n-N+1]]
$$

Vector $y[n]$ is then despread using the filters matched to the different spreading codes to produce an estimate of the $m$ th transmitted symbol

$$
\hat{d}_{k}[m]=\hat{d}_{k}[n N]=c_{k}^{H} y[n N]
$$

where we assume that the spreading sequences have been normalised, e.g. $c_{k}^{H} c_{k}=1$.

In training mode, an error signal for the $m$ th transmitted symbol of the $k$ th user can be obtained as follows:

$$
\begin{aligned}
e_{k}[m] & =d_{k}[m]-\hat{d}_{k}[m] \\
& =d_{k}[m]-c_{k}^{H} X[n N] h^{*}[n N]
\end{aligned}
$$

The criterion for calculating the filter weights $h[n]$ in a $K$ user system is to minimise the following cost function

$$
\begin{aligned}
\xi_{\mathrm{eq}} & =\mathrm{E}\left\{\sum_{k=0}^{K-1} \cdot e_{k}^{2}[m]\right\} \\
& =\mathrm{E}\left\{\sum_{k=0}^{K-1}\left(d_{k}[m]-c_{k}^{H} X[n N] h^{*}[n N]\right)^{2}\right\}
\end{aligned}
$$

An adaptive solution is chosen, which minimises the instantaneous squared error instead of the MSE, leading to

$$
K-1
$$

$$
h[n N]=h[(n-1) N]+2 \mu_{e q} X[n N] \sum_{k=0} e_{k}^{*}[n N] c_{k}^{*}
$$

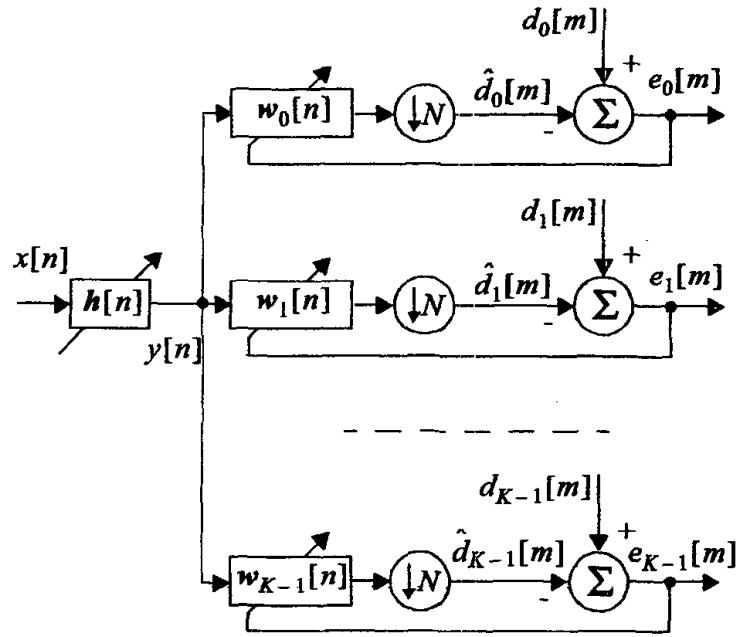

Figure 5: Equaliser and bank of MMSE receivers

where $\mu_{e q}$ is the equaliser step size. Eq. 17 constitutes the update expression of a multiple error filtered-X LMS algorithm [11].

\subsection{Equaliser and Bank of MMSE Receivers}

The third proposed structure, shown in Figure 5, is a modification of the setup of Figure 4. Knowledge of the spreading codes $c_{k}$ is not assumed and adaptive MMSE receivers are used instead of the matched filters. Under these conditions, the weight update expression for the $k$ th adaptive receiver using the NLMS algorithm is

$$
w_{k}[m+1]=w_{k}[m]+2 \frac{\mu}{y^{H}[m] y[m]} e_{k}^{*}[m] y[m]
$$

In total we are updating $K+1$ filters (Eq. 18 for the $K$ filters together with Eq. 17 for the equaliser) with only $K$ error signals. Therefore there is at least one degree of freedom left. To fix this degree of freedom, an additional constraint has to be invoked. In this paper, the following condition is enforced once the update of Eq. 18 has been performed

$$
w_{k}^{H}[m+1] w_{k}[m+1]=1
$$

\section{Simulation}

\subsection{System Setup}

The general simulation setup is shown in Figure 6. A base station transmits data corresponding to $K=3$ channels assigned to one user in a UTRA TDD system with a spreading gain of $Q=N=16$. No other users are assumed to be present in the system. Data symbols at a rate 


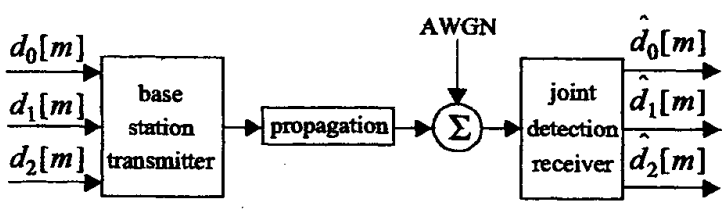

Figure 6: General simulation setup

of $R_{d}=240 \times 10^{3}$ symbols $/$ second are spread using the channelisation and scrambling codes specified in [2] at a rate of $R_{c}=3.84 \times 10^{6} \mathrm{chips} / \mathrm{second}$. Scrambling code 0 is cell specific and therefore used in all 3 channels. Channelisation codes 1 to 3 are applied to spread the spectrum of the data.

The spread spectrum signal is organised in data bursts of type 1 and transmitted through $a$ baseband equivalent multipath Rayleigh fading channel. Two propagation conditions taken from [12] are considered, with their characteristics shown in Table 1. A classic Doppler spectrum is introduced using a carrier frequency of $f_{c}=2.14 \mathrm{GHz}$. Additive white Gaussian noise (AWGN) is applied before the receiver at different levels of chip energy to noise spectral density ratio $E_{c} / N_{0}=\{-10,-5,0,5,10\} \quad \mathrm{dB}$.

\begin{tabular}{|c|c|c|c|}
\hline \multicolumn{2}{|c|}{$\begin{array}{c}\text { ENVIRONMENT A, } \\
\text { speed } 3 \mathrm{~km} / \mathrm{h}\end{array}$} & \multicolumn{2}{c|}{$\begin{array}{r}\text { ENVIRONMENT B, } \\
\text { speed } 3 \mathrm{~km} / \mathrm{h}\end{array}$} \\
\hline $\begin{array}{c}\text { Relative } \\
\text { delay (nsec) }\end{array}$ & $\begin{array}{c}\text { Average } \\
\text { power (dB) }\end{array}$ & $\begin{array}{c}\text { Relative } \\
\text { delay (nsec) }\end{array}$ & $\begin{array}{c}\text { Average } \\
\text { power (dB) }\end{array}$ \\
\hline \hline 0 & 0 & 0 & 0 \\
\hline 976 & -10 & 260 & -3 \\
\hline & & 521 & -6 \\
\hline & 781 & -9 \\
\hline
\end{tabular}

Table 1: Multipath propagation environments

The three structures shown in Figure 3, Figure 4 and Figure 5 are implemented at the receiver. The MMSE receivers operate with $N=16$ coefficients, while the equaliser has a length of $M=31$ weights. By using the midamble of the time bursts the adaptive structures are trained, and the filter coefficients are fixed when the data fields are being decoded. BER error rate values are obtained for the $K=3$ considered channels as a function of $E_{c} / N_{0}$.

\subsection{Simulation Results}

Figure 7 show the BER results as a function of $E_{c} / N_{0}$ for the three considered channels belonging to the same user and decoded with the three presented structures when operating under propagation environment $A$. The results

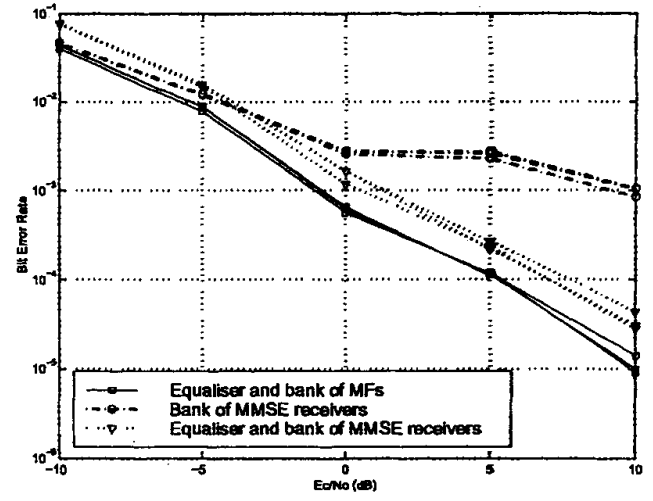

Figure 7: BER vs. $E_{c} / N_{0}$ for the proposed structures when operating under propagation environment A

when operating under environment $B$ are shown in Figure 8.

In both figures it can be seen that noise degrades the performance of all three structures, due to their adaptive nature. For all three structures, the performance under environment $B$ is generally better than under environment A. This is a result of their ability to exploit multipath diversity.

It can be seen that for both propagation environments, the bank of MMSE receivers provide the poorest performance. Although this structure can be considered as a joint detector in the sense that multiple channels are decoded, each channel is despread independently with no information fed between the different branches. This results in a situation where each branch sees the other two channels as interference.

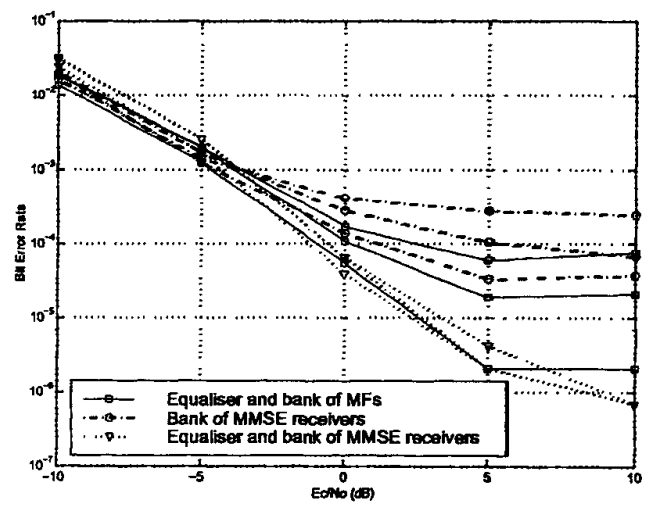

Figure 8: BER vs. $E_{c} / N_{0}$ for the proposed structures when operating under propagation environment $B$ 
For a simple environment such as environment $A$, the three structures exhibit low variability in error rates between different channels. However, for environment B, only the combination of the equaliser and bank of MMSE receivers can retain this desirable quality. By considering the worst of the three channels for each structure, the equaliser and bank of MMSE receivers has a considerably better performance at low noise levels as can be seen in Figure 8.

\section{Conclusions}

We have presented and tested three joint detection structures operating in a UTRA TDD environment under various Rayleigh multipath fading propagation conditions. All the structures attempt to reduce the MAI that appears due to the loss of orthogonality as a result of propagation through a multipath environment.

Of all the tested structures, only the equaliser and bank of MMSE receivers maintains a uniform level of performance across the three channels decoded.

It can also be concluded that the structures which combine information from each branch give a better performance. On this basis the bank of MMSE receivers presents the poorest BER values.

\section{References}

[1] 3G TS 25.201, v.3.0.0, "Physical layer - General description". Oct. 1999.

[2] 3G TS 25.223, v.3.0.0, "Spreading and modulation (TDD)". Oct. 1999.

[3] D. Garcia-Alis, R. W. Stewart. "Implementation of Adaptive Multiuser Receivers for W-CDMA", Digest of IEE Colloquium on Novel DSP Algorithms and Architectures for Radio Systems, London, UK, Sep. 1999.

[4] G. Wootward and B.S. Vucetic, "Adaptive detection for DSCDMA". Proceedings of the IEEE. Vol. 86, No. 7, pp. 14131434, Jul. 1998.

[5] D. Garcia-Alis, R. W. Stewart, "Multiple Code Adaptive Despreader for UTRA TDD", Proceedings of the International Conference on $3 \mathrm{G} 2000$ Mobile Communications Technologies, London, UK, Mar. 2000, pp. 94-98

[6] 3G TS 25.221, v. 3.0.0, "Physical channels and mapping of transpart channels onto physical channels (TDD)". Oct. 1999.

[7] G. L. Stüber, Principles of Mobile Communications. Kluwer Academic Publishers, The Netherlands, 1996.

[8] A. J. Viterbi, CDMA: Principles of Spread Spectrum Communication. Addison Wesley, 1995.

[9] F. Adachi, M. Sawahashi, and H. Suda, "Wideband DSCDMA for Next-Generation mobile communications systems", EEE Comms. Mag. Vol. 36, No. 9, pp. 56-69, Sep. 1998.

[10] S. Haykin, Adaptive Filter Theory, Prentice Hall, 1996.

[11] S.J. Elliott, I.M. Stothers, P.A. Nelson. "A multiple Error
LMS Algorithm and its Applications to the Active Control of Sound and Vibration", IEEE Trans. ASSP, 35(10):14231434, Oct. 1987.

[12] 3G TS 25.105, v. 3.0.0, Oct. 99, "UTRA (BS) TDD; Radio transmission and reception". 